\title{
Expanding the supramolecular toolkit: computed molecular and crystal properties for supporting the crystal engineering of higher- order molecular ionic cocrystals
}

Tamador Alkhidir, $¥$ Zeinab M. Saeed, ${ }^{\ddagger}$ Abeer F. Shunnar, Eman Abujami, Runyararo M. Nyadzayo, Bhausaheb Dhokale and Sharmarke Mohamed*

Department of Chemistry, Green Chemistry \& Materials Modelling Laboratory, Khalifa University of Science and Technology, P.O. Box 127788, Abu Dhabi, United Arab Emirates.

* Corresponding Author Contact Information: Prof. Sharmarke Mohamed - Department of Chemistry, Green Chemistry \& Materials Modelling Laboratory, Khalifa University of Science and Technology, P.O. Box 127788, Abu Dhabi, United Arab Emirates; https://orcid.org/0000-0002-5195-2533; E-mail: sharmarke.mohamed@ku.ac.ae.

‡ Author Contributions: TA and ZMS contributed equally. SM conceived and directed the research. All authors contributed to the analysis of the data and the preparation of the manuscript. 


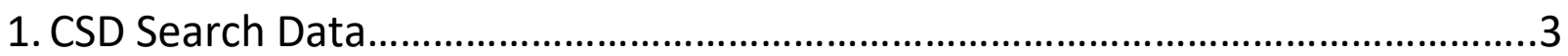

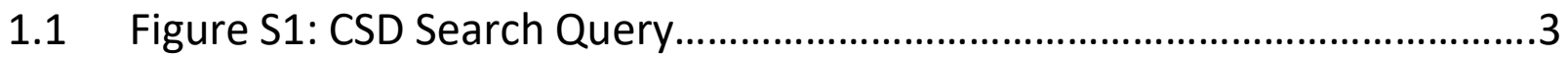

1.2 Table S1: List of Molecular Ionic Cocrystals (ICCS) retrieved from the CSD and their structural properties ........................................................ 4

2. Calculated Molecular and Crystal Properties ............................................ 12

2.1 Table S2: Molecular Properties ......................................................... 12

2.2 Figure S2: Molecular structures for the most common acidic molecules used to crystallize molecular ICCs ....................................................... 16

2.3 Figure S3: Molecular structures for the most common basic molecules used to crystallize molecular ICCs ............................................................. 17

2.4 Table S3: Periodic DFT-D stabilization energies for the molecular ICCs ... 18

2.5 Table S4: Calculated changes in the crystal packing index upon switching

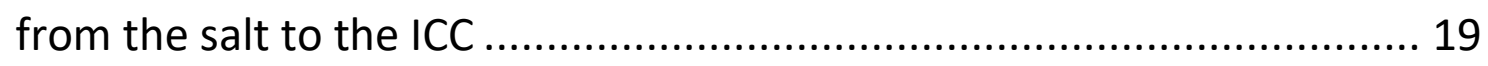

2.6 Table S5: Common motifs found in the molecular ICCs........................ 20 


\section{CSD Search Data}

\subsection{Figure S1: CSD Search Query}

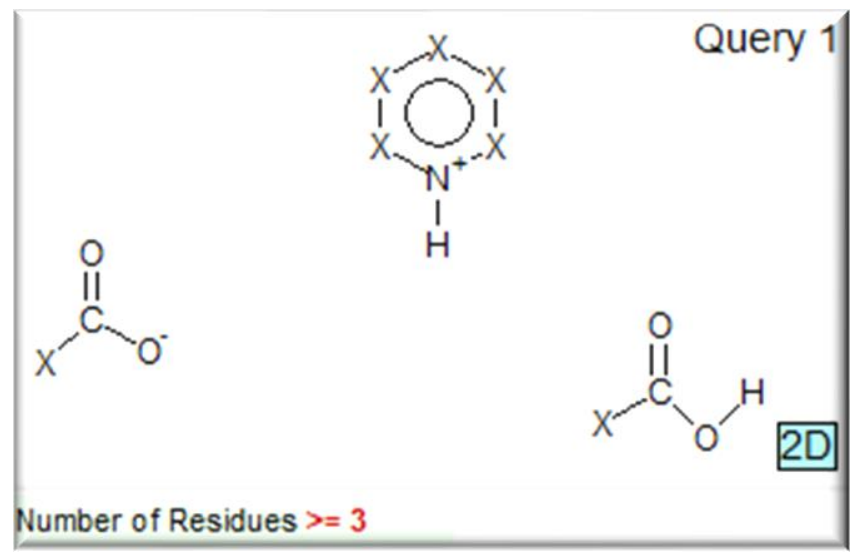

Figure S1: CSD search query for molecular ICCs comprising the following fragments: a protonated six-membered aromatic $\mathrm{N}$-heterocycle, a carboxylate anion and a carboxylic acid fragment. The number of residues in the crystals were set to be equal to or greater than 3. For full details of the search parameters used including the search filters, see the methodology section in the manuscript. 
1.2 Table S1: List of Molecular lonic Cocrystals (ICCS) retrieved from the CSD and their structural properties

\begin{tabular}{|c|c|c|c|c|c|c|c|}
\hline $\begin{array}{l}\text { Crystal } \\
\text { Index }\end{array}$ & $\begin{array}{c}\text { ICC } \\
\text { Refcode }\end{array}$ & CSD Chemical Composition & Solid Form Name & CSD Z' & $\begin{array}{l}\text { CSD } \\
\text { Chemical } \\
\text { Units }\end{array}$ & $\begin{array}{c}N_{C U} \\
\text { (Order) }\end{array}$ & Type of ICC \\
\hline 1 & AFORIY01 & $\begin{array}{c}\left(\mathrm{C}_{13} \mathrm{H}_{11} \mathrm{~N}_{2}\right)^{1+},\left(\mathrm{C}_{7} \mathrm{H}_{4} \mathrm{~N}_{2} \mathrm{O}_{6}\right) \\
\left(\mathrm{C}_{7} \mathrm{H}_{3} \mathrm{~N}_{2} \mathrm{O}_{6}\right)^{1-}\end{array}$ & $\begin{array}{l}\text { 9-Aminoacridinium 2,4-dinitrobenzoate 2,4- } \\
\text { dinitrobenzoic acid }\end{array}$ & 1 & 3 & 3 & $\mathrm{CAB}$ \\
\hline 2 & AJEBIA & $\begin{array}{c}\left(\mathrm{C}_{5} \mathrm{H}_{6} \mathrm{~N}_{1}\right)^{1+},\left(\mathrm{C}_{7} \mathrm{H}_{3} \mathrm{~N}_{2} \mathrm{O}_{7}\right)^{1-} \\
\left(\mathrm{C}_{7} \mathrm{H}_{4} \mathrm{~N}_{2} \mathrm{O}_{7}\right)\end{array}$ & $\begin{array}{l}\text { Pyridinium 3,5-dinitrosalicylate 3,5- } \\
\text { dinitrosalicylic acid }\end{array}$ & 1 & 3 & 3 & CAB \\
\hline 3 & APURAG & $\begin{array}{c}\left(\mathrm{C}_{7} \mathrm{H}_{5} \mathrm{~N}_{1} \mathrm{O}_{4}\right),\left(\mathrm{C}_{7} \mathrm{H}_{4} \mathrm{~N}_{1} \mathrm{O}_{4}\right)^{1-} \\
\left(\mathrm{C}_{6} \mathrm{H}_{8} \mathrm{~N}_{1}\right)^{1+}\end{array}$ & $\begin{array}{l}\text { 3-methylpyridin-1-ium 4-nitrobenzoate 4- } \\
\text { nitrobenzoic acid }\end{array}$ & 1 & 3 & 3 & CAB \\
\hline 4 & AYUSIX & $\begin{array}{c}\left(\mathrm{C}_{8} \mathrm{H}_{12} \mathrm{~N}_{1}\right)^{1+},\left(\mathrm{C}_{7} \mathrm{H}_{5} \mathrm{~N}_{1} \mathrm{O}_{4}\right) \\
\left(\mathrm{C}_{7} \mathrm{H}_{4} \mathrm{~N}_{1} \mathrm{O}_{4}\right)^{1-}\end{array}$ & $\begin{array}{l}\text { 2,4,6-Trimethylpyridinium 4-nitrobenzoate } \\
\text { 4-nitrobenzoic acid }\end{array}$ & 2 & 3 & 3 & $\mathrm{CAB}$ \\
\hline 5 & BEYZAI & $\begin{array}{l}\left(\mathrm{C}_{10} \mathrm{H}_{9} \mathrm{~N}_{2}\right)^{1+},\left(\mathrm{C}_{7} \mathrm{H}_{4} \mathrm{~N}_{2} \mathrm{O}_{6}\right) \\
\left(\mathrm{C}_{7} \mathrm{H}_{3} \mathrm{~N}_{2} \mathrm{O}_{6}\right)^{1-},\left(\mathrm{C}_{7} \mathrm{H}_{7} \mathrm{~N}_{1} \mathrm{O}_{3}\right) \\
\end{array}$ & $\begin{array}{c}\text { 4,4'-bipyridinium 3,5-dinitrobenzoic acid 4- } \\
\text { aminosalicylic acid 3,5-dinitrobenzoate }\end{array}$ & 1 & 4 & 4 & NCAB* \\
\hline 6 & BEYZIQ & $\begin{array}{c}\left(\mathrm{C}_{10} \mathrm{H}_{10} \mathrm{~N}_{2}\right)^{2+}, \\
\left(\mathrm{C}_{10} \mathrm{H}_{8} \mathrm{~N}_{2}\right), 2\left(\mathrm{C}_{7} \mathrm{H}_{4} \mathrm{~N}_{2} \mathrm{O}_{6}\right) \\
2\left(\mathrm{C}_{7} \mathrm{H}_{3} \mathrm{~N}_{2} \mathrm{O}_{6}\right)^{1-}, 2\left(\mathrm{C}_{7} \mathrm{H}_{7} \mathrm{~N}_{1} \mathrm{O}_{2}\right)\end{array}$ & $\begin{array}{c}\text { 4,4'-bipyridinium bis(3,5-dinitrobenzoic acid) 4,4'- } \\
\text { bipyridine bis(4-aminobenzoic acid) bis(3,5- } \\
\text { dinitrobenzoate) }\end{array}$ & 1 & 5 & 5 & NCAB* \\
\hline 7 & CAYJAO01 & $\begin{array}{c}\left(\mathrm{C}_{5} \mathrm{H}_{6} \mathrm{~N}_{1}\right)^{1+},\left(\mathrm{C}_{7} \mathrm{H}_{3} \mathrm{~N}_{2} \mathrm{O}_{6}\right)^{1-} \\
\left(\mathrm{C}_{7} \mathrm{H}_{4} \mathrm{~N}_{2} \mathrm{O}_{6}\right)\end{array}$ & $\begin{array}{l}\text { Pyridinium 3,5-dinitrobenzoate 3,5- } \\
\text { dinitrobenzoic acid }\end{array}$ & 1 & 3 & 3 & $\mathrm{CAB}$ \\
\hline 8 & CIDCUO & $\begin{array}{c}\left(\mathrm{C}_{6} \mathrm{H}_{10} \mathrm{~N}_{3} \mathrm{O}_{2}\right)^{1+},\left(\mathrm{C}_{6} \mathrm{H}_{9} \mathrm{~N}_{3} \mathrm{O}_{2}\right) \\
\left(\mathrm{C}_{4} \mathrm{H}_{5} \mathrm{O}_{6}\right)^{1-}\end{array}$ & $\begin{array}{l}\text { 2-Amino-4,6-dimethoxypyrimidinium hydrogen L- } \\
\text { tartrate 2-amino-4,6-dimethoxypyrimidine }\end{array}$ & 1 & 3 & 3 & $\mathrm{CAB}$ \\
\hline 9 & CUCSEY01 & $\begin{array}{c}\left(\mathrm{C}_{14} \mathrm{H}_{19} \mathrm{~N}_{4} \mathrm{O}_{3}\right)^{1+},\left(\mathrm{C}_{7} \mathrm{H}_{5} \mathrm{O}_{2}\right)^{1-} \\
\left(\mathrm{C}_{7} \mathrm{H}_{6} \mathrm{O}_{2}\right)\end{array}$ & $\begin{array}{l}\text { 2,4-Diamino-5-(3,4,5- } \\
\text { trimethoxybenzyl)pyrimidinium benzoate benzoic } \\
\text { acid }\end{array}$ & 2 & 3 & 3 & $\mathrm{CAB}$ \\
\hline 10 & CUCSEY10 & $\begin{array}{c}\left(\mathrm{C}_{14} \mathrm{H}_{19} \mathrm{~N}_{4} \mathrm{O}_{3}\right)^{1+},\left(\mathrm{C}_{7} \mathrm{H}_{5} \mathrm{O}_{2}\right)^{1-} \\
\left(\mathrm{C}_{7} \mathrm{H}_{6} \mathrm{O}_{2}\right)\end{array}$ & $\begin{array}{l}\text { 2,4-Diamino-5-(3,4,5-trimethoxybenzyl)- } \\
\text { pyrimidinium benzoate benzoic acid solvate }\end{array}$ & 1 & 3 & 3 & $\mathrm{CAB}$ \\
\hline 11 & CUKNON & $\begin{array}{c}\left(\mathrm{C}_{7} \mathrm{H}_{11} \mathrm{~N}_{2}\right)^{1+},\left(\mathrm{C}_{7} \mathrm{H}_{5} \mathrm{Cl}_{1} \mathrm{O}_{2}\right) \\
\left(\mathrm{C}_{7} \mathrm{H}_{4} \mathrm{Cl}_{1} \mathrm{O}_{2}\right)^{1-}\end{array}$ & $\begin{array}{l}\text { 4-(Dimethylamino)pyridinium 4-chlorobenzoate } \\
\text { 4-chlorobenzoic acid }\end{array}$ & 1 & 3 & 3 & $\mathrm{CAB}$ \\
\hline
\end{tabular}




\begin{tabular}{|c|c|c|c|c|c|c|c|}
\hline 12 & CUKNUT & $\begin{array}{c}\left(\mathrm{C}_{7} \mathrm{H}_{11} \mathrm{~N}_{2}\right)^{1+},\left(\mathrm{C}_{7} \mathrm{H}_{6} \mathrm{O}_{3}\right) \\
\left(\mathrm{C}_{7} \mathrm{H}_{5} \mathrm{O}_{3}\right)^{1-}\end{array}$ & $\begin{array}{c}\text { 4-(Dimethylamino)pyridinium 4- } \\
\text { hydroxybenzoate 4-hydroxybenzoic acid }\end{array}$ & 1 & 3 & 3 & $\mathrm{CAB}$ \\
\hline 13 & DEFCUM & $\begin{array}{c}2\left(\mathrm{C}_{5} \mathrm{H}_{6} \mathrm{~N}_{1}\right)^{1+},\left(\mathrm{C}_{2} \mathrm{O}_{4}\right)^{2-} \\
\left(\mathrm{C}_{2} \mathrm{H}_{2} \mathrm{O}_{4}\right)\end{array}$ & bis(Pyridinium) oxalate oxalic acid & 0.5 & 3 & 3 & $C A B$ \\
\hline 14 & DEMYAV & $\begin{array}{c}\left(\mathrm{C}_{17} \mathrm{H}_{24} \mathrm{~N}_{4} \mathrm{O}_{1}\right)^{2+},\left(\mathrm{C}_{2} \mathrm{O}_{4}\right)^{2-} \\
\left(\mathrm{C}_{2} \mathrm{H}_{2} \mathrm{O}_{4}\right)\end{array}$ & $\begin{array}{l}\text { 3-Morpholinium-ethylamino-5-methyl-6- } \\
\text { phenylpyridazinium oxalate oxalic acid }\end{array}$ & 1 & 3 & 3 & $\mathrm{CAB}$ \\
\hline 15 & DICYIA & $\begin{array}{c}2\left(\mathrm{C}_{5} \mathrm{H}_{7} \mathrm{~N}_{2}\right)^{1+},\left(\mathrm{C}_{4} \mathrm{H}_{4} \mathrm{O}_{4}\right)^{2-} \\
\left(\mathrm{C}_{4} \mathrm{H}_{6} \mathrm{O}_{4}\right)\end{array}$ & $\begin{array}{l}\text { bis(2-aminopyridin-1-ium) succinate } \\
\text { succinic acid }\end{array}$ & 0.5 & 3 & 3 & $\mathrm{CAB}$ \\
\hline 16 & DUPJEE & $\begin{array}{c}\left(\mathrm{C}_{5} \mathrm{H}_{8} \mathrm{~N}_{3}\right)^{1+},\left(\mathrm{C}_{7} \mathrm{H}_{5} \mathrm{O}_{2}\right)^{1-} \\
\left(\mathrm{C}_{7} \mathrm{H}_{6} \mathrm{O}_{2}\right)\end{array}$ & $\begin{array}{l}\text { 2,3-Diaminopyridinium benzoate benzoic } \\
\text { acid solvate }\end{array}$ & 1 & 3 & 3 & $C A B$ \\
\hline 17 & DUTNUC & $\begin{array}{c}2\left(\mathrm{C}_{6} \mathrm{H}_{9} \mathrm{~N}_{2}\right)^{1+},\left(\mathrm{C}_{4} \mathrm{H}_{4} \mathrm{O}_{4}\right) \\
\left(\mathrm{C}_{4} \mathrm{H}_{2} \mathrm{O}_{4}\right)^{2-}\end{array}$ & $\begin{array}{l}\text { bis(2-Amino-5-methylpyridinium) } \\
\text { fumarate fumaric acid }\end{array}$ & 0.5 & 3 & 3 & $C A B$ \\
\hline 18 & DUVLUB & $\begin{array}{c}2\left(\mathrm{C}_{5} \mathrm{H}_{6} \mathrm{~N}_{1}\right)^{1+}, 2\left(\mathrm{C}_{2} \mathrm{H}_{1} \mathrm{O}_{4}\right)^{1-} \\
\left(\mathrm{C}_{2} \mathrm{H}_{2} \mathrm{O}_{4}\right)\end{array}$ & $\begin{array}{c}\text { Dipyridinium bis(hydrogen-oxalate) oxalic } \\
\text { acid }\end{array}$ & 0.5 & 3 & 3 & $C A B$ \\
\hline 19 & ECESAJ & $\begin{array}{c}\left(\mathrm{C}_{9} \mathrm{H}_{8} \mathrm{~N}_{1} \mathrm{O}_{4}\right)^{1-},\left(\mathrm{C}_{9} \mathrm{H}_{8} \mathrm{~N}_{1}\right)^{1+} \\
\left(\mathrm{C}_{9} \mathrm{H}_{7} \mathrm{~N}_{1}\right)\end{array}$ & $\begin{array}{c}\text { isoquinolinium [(2- } \\
\text { carboxyphenyl)amino]acetate isoquinoline }\end{array}$ & 1 & 3 & 3 & $\mathrm{CAB}$ \\
\hline 20 & EMUMIK & $\begin{array}{c}\left(\mathrm{C}_{6} \mathrm{H}_{8} \mathrm{~N}_{1}\right)^{1+},\left(\mathrm{C}_{8} \mathrm{H}_{5} \mathrm{O}_{4}\right)^{1-} \\
0.5\left(\mathrm{C}_{8} \mathrm{H}_{6} \mathrm{O}_{4}\right)\end{array}$ & $\begin{array}{l}\text { 2-Methylpyridinium 2-carboxybenzoate } \\
\text { hemikis(benzene-1,2-dicarboxylic acid) }\end{array}$ & 1 & 3 & 3 & $\mathrm{CAB}$ \\
\hline 21 & EYIWOY & $\begin{array}{c}\left(\mathrm{C}_{10} \mathrm{H}_{7} \mathrm{~N}_{1} \mathrm{O}_{2}\right),\left(\mathrm{C}_{10} \mathrm{H}_{8} \mathrm{~N}_{1} \mathrm{O}_{2}\right)^{1+}, \\
\left(\mathrm{C}_{7} \mathrm{H}_{5} \mathrm{O}_{6} \mathrm{~S}_{1}\right)^{1-}\end{array}$ & $\begin{array}{l}\text { Quinolinium-2-carboxylic acid 3-carboxy- } \\
\text { 4-hydroxybenzenesulfonate quinolinium-2- } \\
\text { carboxylate }\end{array}$ & 1 & 3 & 3 & $\mathrm{CAB}$ \\
\hline 22 & EZEGOH & $\begin{array}{c}\left(\mathrm{C}_{8} \mathrm{H}_{11} \mathrm{~N}_{2} \mathrm{~S}_{1}\right)^{1+},\left(\mathrm{C}_{8} \mathrm{H}_{10} \mathrm{~N}_{2} \mathrm{~S}_{1}\right) \\
\left(\mathrm{C}_{7} \mathrm{H}_{4} \mathrm{Cl}_{1} \mathrm{~N}_{1} \mathrm{O}_{4}\right) \\
\left(\mathrm{C}_{7} \mathrm{H}_{3} \mathrm{Cl}_{1} \mathrm{~N}_{1} \mathrm{O}_{4}\right)^{1-}\end{array}$ & $\begin{array}{l}\text { 4-carbamothioyl-2-ethylpyridin-1-ium 2- } \\
\text { ethylpyridine-4-carbothioamide 2-chloro-4- } \\
\text { nitrobenzoic acid 2-chloro-4-nitrobenzoate }\end{array}$ & 1 & 4 & 4 & $C A B$ \\
\hline 23 & FEBSOX & $\begin{array}{c}\left(\mathrm{C}_{6} \mathrm{H}_{7} \mathrm{~N}_{2} \mathrm{O}_{1}\right)^{1+},\left(\mathrm{C}_{7} \mathrm{H}_{4} \mathrm{~N}_{1} \mathrm{O}_{4}\right)^{1-} \\
\left(\mathrm{C}_{6} \mathrm{H}_{6} \mathrm{~N}_{2} \mathrm{O}_{1}\right),\left(\mathrm{C}_{7} \mathrm{H}_{5} \mathrm{~N}_{1} \mathrm{O}_{4}\right)\end{array}$ & $\begin{array}{c}\text { 4-carbamoylpyridin-1-ium 2- } \\
\text { nitrobenzoate pyridine-4-carboxamide 2- } \\
\text { nitrobenzoic acid }\end{array}$ & 1 & 4 & 4 & $C A B$ \\
\hline
\end{tabular}




\begin{tabular}{|c|c|c|c|c|c|c|c|}
\hline 24 & FECGIG & $\begin{array}{c}\left(\mathrm{C}_{6} \mathrm{H}_{7} \mathrm{~N}_{2} \mathrm{O}_{1}\right)^{1+},\left(\mathrm{C}_{7} \mathrm{H}_{5} \mathrm{~N}_{1} \mathrm{O}_{4}\right) \\
\left(\mathrm{C}_{7} \mathrm{H}_{4} \mathrm{~N}_{1} \mathrm{O}_{4}\right)^{1-}\end{array}$ & $\begin{array}{l}\text { 3-carbamoylpyridin-1-ium 2-nitrobenzoic } \\
\text { acid 2-nitrobenzoate }\end{array}$ & 1 & 3 & 3 & $C A B$ \\
\hline 25 & FIBHOP & $\begin{array}{c}\left(\mathrm{C}_{12} \mathrm{H}_{12} \mathrm{~N}_{2}\right)^{2+},\left(\mathrm{C}_{4} \mathrm{H}_{4} \mathrm{O}_{4}\right) \\
\left(\mathrm{C}_{4} \mathrm{H}_{2} \mathrm{O}_{4}\right)^{2-}\end{array}$ & $\begin{array}{l}\text { 2,2'-ethene-1,2-diyldipyridinium but-2- } \\
\text { enedioate but-2-enedioic acid solvate }\end{array}$ & 0.5 & 3 & 3 & $C A B$ \\
\hline 26 & GEPFIR & $\begin{array}{c}\left(\mathrm{C}_{7} \mathrm{H}_{11} \mathrm{~N}_{2}\right)^{1+},\left(\mathrm{C}_{2} \mathrm{H}_{1} \mathrm{Cl}_{2} \mathrm{O}_{2}\right)^{1-} \\
\left(\mathrm{C}_{2} \mathrm{H}_{2} \mathrm{Cl}_{2} \mathrm{O}_{2}\right)\end{array}$ & $\begin{array}{c}\text { 4-(Dimethylamino)pyridinium } \\
\text { dichloroacetate dichloroacetic acid }\end{array}$ & 1 & 3 & 3 & $C A B$ \\
\hline 27 & GIQHUM & $\begin{array}{c}\left(\mathrm{C}_{18} \mathrm{H}_{16} \mathrm{~N}_{3} \mathrm{O}_{1}\right)^{1+},\left(\mathrm{C}_{2} \mathrm{H}_{1} \mathrm{~F}_{3} \mathrm{O}_{2}\right), \\
\left(\mathrm{C}_{2} \mathrm{~F}_{3} \mathrm{O}_{2}\right)^{1-}\end{array}$ & $\begin{array}{c}\text { 3-phenyl-2- } \\
\text { [(phenylcarbamoyl)amino]pyridin-1-ium } \\
\text { trifluoroacetate trifluoroacetic acid }\end{array}$ & 1 & 3 & 3 & CAB \\
\hline 28 & GUKWIT & $\begin{array}{c}2\left(\mathrm{C}_{7} \mathrm{H}_{11} \mathrm{~N}_{2}\right)^{1+},\left(\mathrm{C}_{4} \mathrm{H}_{2} \mathrm{O}_{4}\right)^{2-} \\
\left(\mathrm{C}_{4} \mathrm{H}_{4} \mathrm{O}_{4}\right)\end{array}$ & $\begin{array}{l}\text { bis(4-(Dimethylamino)pyridinium) } \\
\text { fumarate fumaric acid }\end{array}$ & 0.5 & 3 & 3 & $C A B$ \\
\hline 29 & GULRAJ & $\begin{array}{c}\left(\mathrm{C}_{12} \mathrm{H}_{9} \mathrm{~N}_{2}\right)^{1+},\left(\mathrm{C}_{7} \mathrm{H}_{5} \mathrm{~N}_{1} \mathrm{O}_{5}\right) \\
\left(\mathrm{C}_{7} \mathrm{H}_{4} \mathrm{~N}_{1} \mathrm{O}_{5}\right)^{1-}\end{array}$ & $\begin{array}{c}\text { 1,10-phenanthrolin-1-ium (3,6- } \\
\text { dicarboxypyridine 1-oxide) (6-carboxypyridine-3- } \\
\text { carboxylate } 1 \text {-oxide) }\end{array}$ & 1 & 3 & 3 & $C A B$ \\
\hline 30 & HAXFER & $\begin{array}{c}\left(\mathrm{C}_{7} \mathrm{H}_{10} \mathrm{~N}_{1}\right)^{1+},\left(\mathrm{C}_{7} \mathrm{H}_{3} \mathrm{~N}_{2} \mathrm{O}_{6}\right)^{1-} \\
\left(\mathrm{C}_{7} \mathrm{H}_{4} \mathrm{~N}_{2} \mathrm{O}_{6}\right)\end{array}$ & $\begin{array}{c}\text { 3,5-Dimethylpyridinium 3,5- } \\
\text { dinitrobenzoate 3,5-dinitrobenzoic acid }\end{array}$ & 1 & 3 & 3 & $C A B$ \\
\hline 31 & HIFZUU & $\begin{array}{c}\left(\mathrm{C}_{14} \mathrm{H}_{12} \mathrm{O}_{2}\right),\left(\mathrm{C}_{14} \mathrm{H}_{11} \mathrm{O}_{2}\right)^{1-} \\
\left(\mathrm{C}_{5} \mathrm{H}_{7} \mathrm{~N}_{2}\right)^{1+}\end{array}$ & $\begin{array}{l}\text { 2-aminopyridinium diphenyacetate } \\
\text { diphenylacetic acid }\end{array}$ & 1 & 3 & 3 & $C A B$ \\
\hline 32 & IGOGES & $\begin{array}{c}2\left(\mathrm{C}_{14} \mathrm{H}_{12} \mathrm{~N}_{3}\right)^{1+},\left(\mathrm{C}_{8} \mathrm{H}_{6} \mathrm{O}_{4}\right) \\
\left(\mathrm{C}_{8} \mathrm{H}_{4} \mathrm{O}_{4}\right)^{2-}\end{array}$ & $\begin{array}{c}\text { bis }(4-(2-(1 \mathrm{H}-\text { benzimidazol-2- } \\
\text { yl)ethenyl)pyridin-1-ium }) \text { terephthalic acid } \\
\text { terephthalate }\end{array}$ & 0.5 & 3 & 3 & $C A B$ \\
\hline 33 & IHESOD01 & $\begin{array}{c}\left(\mathrm{C}_{4} \mathrm{H}_{6} \mathrm{O}_{4}\right), 2\left(\mathrm{C}_{5} \mathrm{H}_{7} \mathrm{~N}_{2}\right)^{1+} \\
\left(\mathrm{C}_{4} \mathrm{H}_{4} \mathrm{O}_{4}\right)^{2-}\end{array}$ & $\begin{array}{l}\text { bis(2-aminopyridinium) succinic acid } \\
\text { succinate }\end{array}$ & 0.5 & 3 & 3 & $C A B$ \\
\hline 34 & IXOZOL & $\begin{array}{c}\left(\mathrm{C}_{5} \mathrm{H}_{7} \mathrm{~N}_{2}\right)^{1+},\left(\mathrm{C}_{4} \mathrm{H}_{4} \mathrm{O}_{4}\right) \\
\left(\mathrm{C}_{4} \mathrm{H}_{3} \mathrm{O}_{4}\right)^{1-}\end{array}$ & $\begin{array}{l}\text { 4-aminopyridinium hydrogen maleate } \\
\text { maleic acid }\end{array}$ & 1 & 3 & 3 & $C A B$ \\
\hline 35 & JAWVUZ & $\begin{array}{c}\left(\mathrm{C}_{6} \mathrm{H}_{7} \mathrm{~N}_{2} \mathrm{O}_{1}\right)^{1+},\left(\mathrm{C}_{6} \mathrm{H}_{6} \mathrm{~N}_{2} \mathrm{O}_{1}\right) \\
\left(\mathrm{C}_{4} \mathrm{H}_{5} \mathrm{O}_{6}\right)^{1-} \\
\end{array}$ & $\begin{array}{l}\text { Pyridinium-4-carboxamide pyridine-4- } \\
\text { carboxamide D-(+)-hydrogen tartrate }\end{array}$ & 1 & 3 & 3 & $C A B$ \\
\hline 36 & JEJNAO & $\begin{array}{c}\left(\mathrm{C}_{10} \mathrm{H}_{9} \mathrm{~N}_{2}\right)^{1+},\left(\mathrm{C}_{7} \mathrm{H}_{3} \mathrm{~N}_{2} \mathrm{O}_{6}\right)^{1-} \\
\left(\mathrm{C}_{7} \mathrm{H}_{4} \mathrm{~N}_{2} \mathrm{O}_{6}\right)\end{array}$ & $\begin{array}{l}\text { 2,2'-Bipyridinium 3,5-dinitrobenzoate 3,5- } \\
\text { dinitrobenzoic acid }\end{array}$ & 1 & 3 & 3 & $C A B$ \\
\hline 37 & JOYJIS & $\begin{array}{c}2\left(\mathrm{C}_{5} \mathrm{H}_{8} \mathrm{O}_{4}\right),\left(\mathrm{C}_{5} \mathrm{H}_{6} \mathrm{O}_{4}\right) 2- \\
2\left(\mathrm{C}_{4} \mathrm{H}_{6} \mathrm{~N}_{3}\right)^{1+} \\
\end{array}$ & $\begin{array}{l}\text { bis(2-aminopyrimidin-1-ium) bis(glutaric } \\
\text { acid) glutarate }\end{array}$ & 1 & 3 & 3 & $C A B$ \\
\hline 38 & KALQUM & $\begin{array}{c}\left(\mathrm{C}_{6} \mathrm{H}_{7} \mathrm{~N}_{2} \mathrm{O}_{1}\right)^{1+},\left(\mathrm{C}_{6} \mathrm{H}_{6} \mathrm{~N}_{2} \mathrm{O}_{1}\right) \\
\left(\mathrm{C}_{4} \mathrm{H}_{5} \mathrm{O}_{5}\right)^{1-}\end{array}$ & $\begin{array}{l}\text { 4-carbamoylpyridin-1-ium pyridine-4- } \\
\text { carboxamide 3-carboxy-2-hydroxypropanoate }\end{array}$ & 1 & 3 & 3 & $C A B$ \\
\hline
\end{tabular}




\begin{tabular}{|c|c|c|c|c|c|c|c|}
\hline 39 & KEKHAK & $\begin{array}{c}\left(\mathrm{C}_{10} \mathrm{H}_{7} \mathrm{~N}_{1} \mathrm{O}_{4}\right),\left(\mathrm{C}_{10} \mathrm{H}_{6} \mathrm{~N}_{1} \mathrm{O}_{4}\right)^{1-} \\
\left(\mathrm{C}_{9} \mathrm{H}_{8} \mathrm{~N}_{1} \mathrm{O}_{1}\right)^{1+}\end{array}$ & $\begin{array}{l}\text { 8-Hydroxyquinolinium phthaloylglycinate } \\
\text { phthaloylglycine }\end{array}$ & 1 & 3 & 3 & $\mathrm{CAB}$ \\
\hline 40 & KOXREU & $\begin{array}{c}\left(\mathrm{C}_{3} \mathrm{H}_{3} \mathrm{O}_{4}\right)^{1-},\left(\mathrm{C}_{3} \mathrm{H}_{4} \mathrm{O}_{4}\right) \\
\left(\mathrm{C}_{5} \mathrm{H}_{6} \mathrm{~N}_{1}\right)^{1+} \\
\end{array}$ & Pyridinium trihydrogen dimalonate & 1 & 3 & 3 & $\mathrm{CAB}$ \\
\hline 41 & KUHTUE & $\begin{array}{c}\left(\mathrm{C}_{7} \mathrm{H}_{11} \mathrm{~N}_{2}\right)^{1+},\left(\mathrm{C}_{7} \mathrm{H}_{5} \mathrm{O}_{3}\right)^{1-} \\
\left(\mathrm{C}_{7} \mathrm{H}_{6} \mathrm{O}_{2}\right)\end{array}$ & $\begin{array}{l}\text { 4-(dimethylamino)pyridinium salicylate } \\
\text { benzoic acid solvate }\end{array}$ & 1 & 3 & 3 & NCAB \\
\hline 42 & KUHVAM & $\begin{array}{c}\left(\mathrm{C}_{7} \mathrm{H}_{11} \mathrm{~N}_{2}\right)^{1+},\left(\mathrm{C}_{7} \mathrm{H}_{5} \mathrm{Cl}_{1} \mathrm{O}_{2}\right) \\
\left(\mathrm{C}_{7} \mathrm{H}_{5} \mathrm{O}_{3}\right)^{1-}\end{array}$ & $\begin{array}{l}\text { 4-(dimethylamino)pyridinium salicylate 4- } \\
\text { chlorobenzoic acid }\end{array}$ & 1 & 3 & 3 & NCAB \\
\hline 43 & KUHVEQ & $\begin{array}{c}\left(\mathrm{C}_{7} \mathrm{H}_{11} \mathrm{~N}_{2}\right)^{1+},\left(\mathrm{C}_{7} \mathrm{H}_{6} \mathrm{O}_{3}\right) \\
\left(\mathrm{C}_{7} \mathrm{H}_{5} \mathrm{O}_{3}\right)^{1-} \\
\end{array}$ & $\begin{array}{l}\text { 4-(dimethylamino)pyridinium salicylate 4- } \\
\text { hydroxybenzoic acid }\end{array}$ & 1 & 3 & 3 & NCAB \\
\hline 44 & KUJDIE & $\begin{array}{c}\left(\mathrm{C}_{7} \mathrm{H}_{11} \mathrm{~N}_{2}\right)^{1+},\left(\mathrm{C}_{7} \mathrm{H}_{6} \mathrm{O}_{3}\right) \\
\left(\mathrm{C}_{7} \mathrm{H}_{5} \mathrm{O}_{3}\right)^{1-}\end{array}$ & $\begin{array}{l}\text { 4-(Dimethylamino)pyridinium salicylate } \\
\text { salicylic acid }\end{array}$ & 1 & 3 & 3 & $\mathrm{CAB}$ \\
\hline 45 & KUZJIZ & $\begin{array}{c}0.33\left(\mathrm{C}_{8} \mathrm{H}_{6} \mathrm{O}_{4}\right),\left(\mathrm{C}_{8} \mathrm{H}_{5} \mathrm{O} 4\right)^{1-} \\
\left(\mathrm{C}_{5} \mathrm{H}_{6} \mathrm{Cl}_{1} \mathrm{~N}_{2}\right)^{1+}\end{array}$ & $\begin{array}{l}\text { 2-Amino-5-chloropyridinium 2- } \\
\text { carboxybenzoate phthalic acid solvate }\end{array}$ & 3 & 3 & 3 & $\mathrm{CAB}$ \\
\hline 46 & LAPNUM & $\begin{array}{c}2\left(\mathrm{C}_{6} \mathrm{H}_{9} \mathrm{~N}_{2}\right)^{1+},\left(\mathrm{C}_{6} \mathrm{H}_{8} \mathrm{O}_{4}\right)^{2-} \\
\left(\mathrm{C}_{6} \mathrm{H}_{10} \mathrm{O}_{4}\right)\end{array}$ & $\begin{array}{l}\text { bis(2-Amino-5-methylpyridinium) adipate } \\
\text { adipic acid }\end{array}$ & 0.5 & 3 & 3 & $\mathrm{CAB}$ \\
\hline 47 & LAPPAU & $\begin{array}{c}2\left(\mathrm{C}_{6} \mathrm{H}_{9} \mathrm{~N}_{2}\right)^{1+},\left(\mathrm{C}_{6} \mathrm{H}_{2} \mathrm{O}_{4} \mathrm{~S}_{1}\right)^{2-} \\
\left(\mathrm{C}_{6} \mathrm{H}_{4} \mathrm{O}_{4} \mathrm{~S}_{1}\right)\end{array}$ & $\begin{array}{l}\text { bis(2-Amino-5-methylpyridinium) } 2,5- \\
\text { thiophenedicarboxylate } 2,5- \\
\text { thiophenedicarboxylic acid }\end{array}$ & 0.5 & 3 & 3 & $\mathrm{CAB}$ \\
\hline 48 & LATLID & $\begin{array}{r}\left(\mathrm{C}_{9} \mathrm{H}_{12} \mathrm{~N}_{3} \mathrm{O}_{1}\right)^{1+} \\
3\left(\mathrm{C}_{9} \mathrm{H}_{11} \mathrm{~N}_{3} \mathrm{O}_{1}\right) \\
3\left(\mathrm{C}_{7} \mathrm{H}_{4} \mathrm{Cl}_{1} \mathrm{~N}_{1} \mathrm{O}_{4}\right) \\
\left(\mathrm{C}_{7} \mathrm{H}_{3} \mathrm{Cl}_{1} \mathrm{~N}_{1} \mathrm{O}_{4}\right)^{1-}\end{array}$ & $\begin{array}{c}\text { N'-Isopropylideneisonicotinohydrazidium } \\
\text { 2-chloro-4-nitrobenzoate tris(N'- } \\
\text { isopropylideneisonicotinohydrazide 2-chloro-4- } \\
\text { nitrobenzoic acid) }\end{array}$ & 1 & 4 & 4 & $\mathrm{CAB}$ \\
\hline 49 & LOCMUM & $\begin{array}{c}2\left(\mathrm{C}_{12} \mathrm{H}_{9} \mathrm{~N}_{2}\right)^{1+},\left(\mathrm{C}_{10} \mathrm{H}_{4} \mathrm{O}_{8}\right)^{2-} \\
\left(\mathrm{C}_{10} \mathrm{H}_{6} \mathrm{O}_{8}\right)\end{array}$ & $\begin{array}{c}\text { bis(1,10-Phenanthrolinium) dihydrogen } \\
\text { benzene-1,2,4,5-tetracarboxylate benzene- } \\
\text { 1,2,4,5-tetracarboxylic acid } \\
\end{array}$ & 0.5 & 3 & 3 & $\mathrm{CAB}$ \\
\hline 50 & LORWOF & $\begin{array}{c}\left(\mathrm{C}_{6} \mathrm{H}_{7} \mathrm{~N}_{2} \mathrm{O}_{1}\right)^{1+},\left(\mathrm{C}_{8} \mathrm{H}_{3} \mathrm{Cl}_{2} \mathrm{O}_{4}\right)^{1-} \\
\left(\mathrm{C}_{9} \mathrm{H}_{6} \mathrm{Cl}_{2} \mathrm{O}_{4}\right)\end{array}$ & $\begin{array}{l}\text { 4-(Carbamoyl)pyridinium 2-carboxy-4,5- } \\
\text { dichlorobenzoate methyl 2-carboxy-4,5- } \\
\text { dichlorobenzoate }\end{array}$ & 1 & 3 & 3 & NCAB \\
\hline 51 & MELZAF & $\begin{array}{c}4\left(\mathrm{C}_{12} \mathrm{H}_{9} \mathrm{~N}_{2}\right)^{1+},\left(\mathrm{C}_{10} \mathrm{H}_{4} \mathrm{O}_{8}\right)^{2-} \\
2\left(\mathrm{C}_{10} \mathrm{H}_{5} \mathrm{O}_{8}\right)^{1-}\end{array}$ & $\begin{array}{l}\text { tetrakis(1,10-Phenanthrolinium) bis(2,4,5- } \\
\text { tricarboxybenzoate) 2,5-dicarboxybenzene-1,4- } \\
\text { dicarboxylate }\end{array}$ & 0.5 & 3 & 3 & CAB \\
\hline
\end{tabular}




\begin{tabular}{|c|c|c|c|c|c|c|c|}
\hline 52 & MEXDAW & $\begin{array}{c}2\left(\mathrm{C}_{10} \mathrm{H}_{11} \mathrm{~N}_{2}\right)^{1+},\left(\mathrm{C}_{3} \mathrm{H}_{4} \mathrm{O}_{4}\right) \\
2\left(\mathrm{C}_{3} \mathrm{H}_{3} \mathrm{O}_{4}\right)^{1-}\end{array}$ & $\begin{array}{l}\text { 5-Amino-6-methylquinolin-1-ium } \\
\text { hydrogen dimalonate malonic acid }\end{array}$ & 0.5 & 3 & 3 & $\mathrm{CAB}$ \\
\hline 53 & MUFJUV & $\begin{array}{c}\left(\mathrm{C}_{7} \mathrm{H}_{11} \mathrm{~N}_{2}\right)^{1+},\left(\mathrm{C}_{7} \mathrm{H}_{5} \mathrm{Cl}_{1} \mathrm{O}_{2}\right) \\
\left(\mathrm{C}_{7} \mathrm{H}_{4} \mathrm{Cl}_{1} \mathrm{O}_{2}\right)^{1-} \\
\end{array}$ & $\begin{array}{c}\text { 4-dimethylaminopyridinium 2- } \\
\text { chlorobenzoate 2-chlorobenzoic acid solvate }\end{array}$ & 1 & 3 & 3 & $\mathrm{CAB}$ \\
\hline 54 & $\begin{array}{l}\text { MUYSOPO } \\
1\end{array}$ & $\begin{array}{l}\left(\mathrm{C}_{12} \mathrm{H}_{7} \mathrm{~F}_{2} \mathrm{~N}_{2} \mathrm{O}_{2}\right)^{1-} \\
3\left(\mathrm{C}_{12} \mathrm{H}_{8} \mathrm{~F}_{2} \mathrm{~N}_{2} \mathrm{O}_{2}\right) \\
\left(\mathrm{C}_{12} \mathrm{H}_{9} \mathrm{~F}_{2} \mathrm{~N}_{2} \mathrm{O}_{2}\right)^{1+} \\
\end{array}$ & $\begin{array}{l}\text { 2-((2,6-Difluorophenyl)amino)nicotinic } \\
\text { acid }\end{array}$ & 1 & 3 & 3 & $\mathrm{CAB}$ \\
\hline 55 & NEWNIP & $\begin{array}{c}\left(\mathrm{C}_{15} \mathrm{H}_{10} \mathrm{O}_{2}\right),\left(\mathrm{C}_{7} \mathrm{H}_{3} \mathrm{~N}_{2} \mathrm{O}_{6}\right)^{1-} \\
\left(\mathrm{C}_{5} \mathrm{H}_{6} \mathrm{Br}_{1} \mathrm{~N}_{2}\right)^{1+}\end{array}$ & $\begin{array}{l}\text { anthracene-9-carboxylic acid 2-amino-3- } \\
\text { bromopyridinium 3,5-dinitrobenzoate }\end{array}$ & 1 & 3 & 3 & NCAB \\
\hline 56 & NIMDIW & $\begin{array}{c}\left(\mathrm{C}_{9} \mathrm{H}_{8} \mathrm{~N}_{1} \mathrm{O}_{1}\right)^{1+},\left(\mathrm{C}_{7} \mathrm{H}_{5} \mathrm{O}_{3}\right)^{1-} \\
\left(\mathrm{C}_{7} \mathrm{H}_{6} \mathrm{O}_{3}\right)\end{array}$ & $\begin{array}{l}\text { 8-Hydroxyquinoline salicylate salicylic } \\
\text { acid }\end{array}$ & 1 & 3 & 3 & CAB \\
\hline 57 & OJUJUA & $\begin{array}{l}\left(\mathrm{C}_{15} \mathrm{H}_{10} \mathrm{O}_{2}\right),\left(\mathrm{C}_{15} \mathrm{H}_{9} \mathrm{O}_{2}\right)^{1-} \\
2\left(\mathrm{C}_{6} \mathrm{H}_{3} \mathrm{~N}_{3} \mathrm{O}_{6}\right),\left(\mathrm{C}_{5} \mathrm{H}_{7} \mathrm{~N}_{2}\right)^{1+}\end{array}$ & $\begin{array}{l}\text { 3-aminopyridin-1-ium anthracene-9- } \\
\text { carboxylate bis(anthracene-9-carboxylic acid) } \\
\text { 1,3,5-trinitrobenzene }\end{array}$ & 1 & 4 & 4 & $\mathrm{CAB}$ \\
\hline 58 & OVUMEY & $\begin{array}{c}\left(\mathrm{C}_{9} \mathrm{H}_{8} \mathrm{Cl}_{2} \mathrm{~N}_{5}\right)^{1+},\left(\mathrm{C}_{9} \mathrm{H}_{7} \mathrm{Cl}_{2} \mathrm{~N}_{5}\right) \\
\left(\mathrm{C}_{7} \mathrm{H}_{4} \mathrm{~F}_{1} \mathrm{O}_{2}\right)^{1-},\left(\mathrm{C}_{7} \mathrm{H}_{5} \mathrm{~F}_{1} \mathrm{O}_{2}\right)\end{array}$ & $\begin{array}{l}\text { 3,5-Diamino-6-(2,3-dichlorophenyl)-1,2,4- } \\
\text { triazin-2-ium 3,5-diamino-6-(2,3-dichlorophenyl)- } \\
\text { 1,2,4-triazine 4-fluorobenzoate 4-fluorobenzoic } \\
\text { acid }\end{array}$ & 1 & 4 & 4 & $\mathrm{CAB}$ \\
\hline 59 & PEKSUV & $\begin{array}{c}\left(\mathrm{C}_{10} \mathrm{H}_{9} \mathrm{~N}_{2}\right)^{1+},\left(\mathrm{C}_{7} \mathrm{H}_{6} \mathrm{O}_{4}\right) \\
\left(\mathrm{C}_{7} \mathrm{H}_{5} \mathrm{O}_{4}\right)^{1-}\end{array}$ & $\begin{array}{c}\text { 4-(pyrid-4-ly)-pyridinium 2,5- } \\
\text { dihydroxybenzoate dihydroxybenzoic acid }\end{array}$ & 1 & 3 & 3 & $\mathrm{CAB}$ \\
\hline 60 & PIWXEA & $\begin{array}{l}2\left(\mathrm{C}_{18} \mathrm{H}_{18} \mathrm{~N}_{3} \mathrm{O}_{3} \mathrm{~S}_{1}\right)^{1+} \\
\left(\mathrm{C}_{8} \mathrm{H}_{14} \mathrm{O}_{4}\right),\left(\mathrm{C}_{8} \mathrm{H}_{12} \mathrm{O}_{4}\right)^{2-}\end{array}$ & $\begin{array}{l}\text { bis(6'-methoxy-5-(4- } \\
\text { (methylsulfonyl)phenyl)-2-amino-3,3'-bipyridin-1- } \\
\text { ium) suberate suberic acid }\end{array}$ & 0.5 & 3 & 3 & $\mathrm{CAB}$ \\
\hline 61 & QABZAX & $\begin{array}{c}\left(\mathrm{C}_{28} \mathrm{H}_{15} \mathrm{~N}_{4}\right)^{1+},\left(\mathrm{C}_{2} \mathrm{~F}_{3} \mathrm{O}_{2}\right)^{1-} \\
\left(\mathrm{C}_{2} \mathrm{H}_{1} \mathrm{~F}_{3} \mathrm{O}_{2}\right)\end{array}$ & $\begin{array}{l}\text { quinoxalino }\left[2^{\prime}, 3^{\prime}: 9,10\right] \text { phenanthro }[4,5- \\
\text { abc]phenazin-1-ium trifluoroacetate } \\
\text { trifluoroacetic acid }\end{array}$ & 1 & 3 & 3 & $\mathrm{CAB}$ \\
\hline 62 & QAFFOS & $\begin{array}{c}\left(\mathrm{C}_{5} \mathrm{H}_{6} \mathrm{~N}_{1}\right)^{1+},\left(\mathrm{C}_{1} \mathrm{H}_{1} \mathrm{O}_{2}\right)^{1-} \\
3\left(\mathrm{C}_{1} \mathrm{H}_{2} \mathrm{O}_{2}\right) \\
\end{array}$ & Pyridinium formate tris(formic acid) & 1 & 3 & 3 & $\mathrm{CAB}$ \\
\hline 63 & $\begin{array}{c}\text { QAFFOSO } \\
1 \\
\end{array}$ & $\begin{array}{c}\left(\mathrm{C}_{5} \mathrm{H}_{6} \mathrm{~N}_{1}\right)^{1+}, 3\left(\mathrm{C}_{1} \mathrm{H}_{2} \mathrm{O}_{2}\right) \\
\left(\mathrm{C}_{1} \mathrm{H}_{1} \mathrm{O}_{2}\right)^{1-} \\
\end{array}$ & Pyridinium formate tris(formic acid) & 1 & 3 & 3 & $\mathrm{CAB}$ \\
\hline
\end{tabular}




\begin{tabular}{|c|c|c|c|c|c|c|c|}
\hline 64 & RENVUE & $\begin{array}{c}\left(\mathrm{C}_{9} \mathrm{H}_{9} \mathrm{~N}_{1} \mathrm{O}_{4}\right),\left(\mathrm{C}_{9} \mathrm{H}_{8} \mathrm{~N}_{1} \mathrm{O}_{4}\right)^{1-} \\
\left(\mathrm{C}_{6} \mathrm{H}_{8} \mathrm{~N}_{1}\right)^{1+}\end{array}$ & $\begin{array}{c}\text { 2-methylpyridinium [(2- } \\
\text { carboxyphenyl)amino]acetate } 2- \\
\text { [(carboxymethyl)amino]benzoic acid }\end{array}$ & 1 & 3 & 3 & CAB \\
\hline 65 & RENWAL & $\begin{array}{c}\left(\mathrm{C}_{9} \mathrm{H}_{9} \mathrm{~N}_{1} \mathrm{O}_{4}\right), 2\left(\mathrm{C}_{9} \mathrm{H}_{8} \mathrm{~N}_{1} \mathrm{O}_{4}\right)^{1-} \\
2\left(\mathrm{C}_{6} \mathrm{H}_{8} \mathrm{~N}_{1}\right)^{1+}\end{array}$ & $\begin{array}{c}\text { 4-methylpyridinium [(2- } \\
\text { carboxyphenyl)amino]acetate } 2- \\
\text { [(carboxymethyl)amino]benzoic acid }\end{array}$ & 1 & 3 & 3 & CAB \\
\hline 66 & RESFUR & $\begin{array}{c}\left(\mathrm{C}_{7} \mathrm{H}_{10} \mathrm{~N}_{1}\right)^{1+}, 0.5\left(\mathrm{C}_{4} \mathrm{H}_{2} \mathrm{O}_{4}\right)^{2-} \\
0.5\left(\mathrm{C}_{4} \mathrm{H}_{4} \mathrm{O}_{4}\right)\end{array}$ & $\begin{array}{l}\text { 2,4-lutidinium hemikis(fumarate fumaric } \\
\text { acid) }\end{array}$ & 1 & 3 & 3 & CAB \\
\hline 67 & RESGEC & $\begin{array}{c}\left(\mathrm{C}_{7} \mathrm{H}_{10} \mathrm{~N}_{1}\right)^{1+}, 0.5\left(\mathrm{C}_{4} \mathrm{H}_{2} \mathrm{O}_{4}\right)^{2-} \\
\left(\mathrm{C}_{4} \mathrm{H}_{4} \mathrm{O} 4\right)\end{array}$ & $\begin{array}{l}\text { 2,5-lutidinium hemikis(fumarate) fumaric } \\
\text { acid }\end{array}$ & 1 & 3 & 3 & CAB \\
\hline 68 & RESGUS & $\begin{array}{c}\left(\mathrm{C}_{7} \mathrm{H}_{10} \mathrm{~N}_{1}\right)^{1+}, 0.5\left(\mathrm{C}_{4} \mathrm{H}_{2} \mathrm{O}_{4}\right)^{2-} \\
\left(\mathrm{C}_{4} \mathrm{H}_{4} \mathrm{O}_{4}\right)\end{array}$ & $\begin{array}{l}\text { 3,4-lutidine hemikis(fumarate) fumaric } \\
\text { acid }\end{array}$ & 1 & 3 & 3 & $C A B$ \\
\hline 69 & REXNOZ & $\begin{array}{c}2\left(\mathrm{C}_{7} \mathrm{H}_{11} \mathrm{~N}_{2}\right)^{1+},\left(\mathrm{C}_{8} \mathrm{H}_{2} \mathrm{~F}_{4} \mathrm{O}_{4}\right) \\
\left(\mathrm{C}_{8} \mathrm{~F}_{4} \mathrm{O}_{4}\right)^{2-}\end{array}$ & $\begin{array}{l}\text { bis(4-(dimethylamino)pyridinium) 2,3,5,6- } \\
\text { tetrafluoroterephthalate } 2,3,5,6 \text { - } \\
\text { tetrafluoroterephthalic acid }\end{array}$ & 0.5 & 3 & 3 & CAB \\
\hline 70 & ROBFAS & $\begin{array}{c}\left(\mathrm{C}_{12} \mathrm{H}_{14} \mathrm{Cl}_{1} \mathrm{~N}_{4}\right)^{1+},\left(\mathrm{C}_{3} \mathrm{H}_{4} \mathrm{O}_{2}\right) \\
\left(\mathrm{C}_{3} \mathrm{H}_{3} \mathrm{O}_{2}\right)^{1-}\end{array}$ & $\begin{array}{l}\text { 2,4-diamino-5-(4-chlorophenyl)-6- } \\
\text { ethylpyrimidin-1-ium prop-2-enoate prop-2-enoic } \\
\text { acid }\end{array}$ & 1 & 3 & 3 & $C A B$ \\
\hline 71 & ROFCEV & $\begin{array}{c}\left(\mathrm{C}_{7} \mathrm{H}_{5} \mathrm{~N}_{1} \mathrm{O}_{4}\right),\left(\mathrm{C}_{7} \mathrm{H}_{4} \mathrm{~N}_{1} \mathrm{O}_{4}\right)^{1-} \\
\left(\mathrm{C}_{5} \mathrm{H}_{7} \mathrm{~N}_{2}\right)^{1+}\end{array}$ & $\begin{array}{l}\text { 4-aminopyridinium 4-nitrobenzoic acid 4- } \\
\text { nitrobenzoate }\end{array}$ & 1 & 3 & 3 & $C A B$ \\
\hline 72 & SAGWII & $\begin{array}{c}\left(\mathrm{C}_{5} \mathrm{H}_{8} \mathrm{~N}_{3}\right)^{1+},\left(\mathrm{C}_{6} \mathrm{H}_{7} \mathrm{O}_{2}\right)^{1-} \\
\left(\mathrm{C}_{6} \mathrm{H}_{8} \mathrm{O}_{2}\right)\end{array}$ & $\begin{array}{l}\text { 2,3-Diaminopyridinium hexa-2,4-dienoic } \\
\text { acid hexa-2,4-dienoate }\end{array}$ & 1 & 3 & 3 & $C A B$ \\
\hline 73 & SEGSAZ & $\begin{array}{c}\left(\mathrm{C}_{17} \mathrm{H}_{27} \mathrm{~N}_{4}\right)^{1+}, 0.5\left(\mathrm{C}_{4} \mathrm{H}_{2} \mathrm{O}_{4}\right)^{2-} \\
\left(\mathrm{C}_{4} \mathrm{H}_{4} \mathrm{O}_{4}\right)\end{array}$ & $\begin{array}{l}\text { 3-Cyclopropylamino-2-cyclopropyl-5- } \\
\text { methyl-6-(azepinyl)pyrimidinium } \\
\text { hemikis(fumarate) fumaric acid solvate }\end{array}$ & 1 & 3 & 3 & $C A B$ \\
\hline 74 & TAKZUC & $\begin{array}{c}\left(\mathrm{C}_{8} \mathrm{H}_{7} \mathrm{O}_{3}\right)^{1-},\left(\mathrm{C}_{8} \mathrm{H}_{8} \mathrm{O}_{3}\right) \\
\left(\mathrm{C}_{9} \mathrm{H}_{9} \mathrm{~N}_{2}\right)^{1+}\end{array}$ & $\begin{array}{l}\text { 1-aminoisoquinolinium } \\
\text { hydroxy(phenyl)acetate hydroxy(phenyl)acetic } \\
\text { acid }\end{array}$ & 1 & 3 & 3 & CAB \\
\hline
\end{tabular}




\begin{tabular}{|c|c|c|c|c|c|c|c|}
\hline 75 & TEKLOK & $\begin{array}{c}2\left(\mathrm{C}_{10} \mathrm{H}_{9} \mathrm{~N}_{2}\right)^{1+},\left(\mathrm{C}_{10} \mathrm{H}_{4} \mathrm{O}_{8}\right)^{2-}, \\
\left(\mathrm{C}_{10} \mathrm{H}_{6} \mathrm{O}_{8}\right)\end{array}$ & $\begin{array}{c}\text { bis(2,2'-Bipyridinium) benzene-1,4- } \\
\text { dicarboxylate-2,5-dicarboxylic acid benzene- } \\
\text { 1,2,4,5-tetracarboxylic acid }\end{array}$ & 0.5 & 3 & 3 & $\mathrm{CAB}$ \\
\hline 76 & TICZOW & $\begin{array}{c}\left(\mathrm{C}_{11} \mathrm{H}_{10} \mathrm{~N}_{1}\right)^{1+},\left(\mathrm{C}_{7} \mathrm{H}_{3} \mathrm{~N}_{2} \mathrm{O}_{6}\right)^{1-} \\
\left(\mathrm{C}_{7} \mathrm{H}_{4} \mathrm{~N}_{2} \mathrm{O}_{6}\right)\end{array}$ & $\begin{array}{l}\text { 4-phenylpyridinium 3,5-dinitrobenzoic } \\
\text { acid 3,5-dinitrobenzoate }\end{array}$ & 1 & 3 & 3 & $\mathrm{CAB}$ \\
\hline 77 & UNECEV & $\begin{array}{c}2\left(\mathrm{C}_{7} \mathrm{H}_{11} \mathrm{~N}_{2}\right)^{1+},\left(\mathrm{C}_{10} \mathrm{H}_{6} \mathrm{O}_{8}\right), \\
\left(\mathrm{C}_{10} \mathrm{H}_{4} \mathrm{O}_{8}\right)^{2-}\end{array}$ & $\begin{array}{c}\text { 1,2,4,5-Benzenetetracarboxylic acid } \\
\text { benzene-2,5-dicarboxylate-1,4-dicarboxylic acid } \\
\text { bis(4-(dimethylamino)pyridinium) clathrate }\end{array}$ & 0.5 & 3 & 3 & $\mathrm{CAB}$ \\
\hline 78 & UVOKIA & $\begin{array}{c}2\left(\mathrm{C}_{6} \mathrm{H}_{9} \mathrm{~N}_{2}\right)^{1+},\left(\mathrm{C}_{8} \mathrm{Cl}_{4} \mathrm{O}_{4}\right)^{2-} \\
\left(\mathrm{C}_{8} \mathrm{H}_{2} \mathrm{Cl}_{4} \mathrm{O}_{4}\right)\end{array}$ & $\begin{array}{l}\text { bis(2-amino-3-methylpyridin-1-ium) } \\
\text { 3,4,5,6-tetrachlorophthalate 3,4,5,6- } \\
\text { tetrachlorophthalic acid }\end{array}$ & 1 & 3 & 3 & $\mathrm{CAB}$ \\
\hline 79 & UYOZEO & $\begin{array}{c}2\left(\mathrm{C}_{10} \mathrm{H}_{9} \mathrm{~N}_{2}\right)^{1+},\left(\mathrm{C}_{8} \mathrm{H}_{2} \mathrm{~F}_{4} \mathrm{O}_{4}\right), \\
\left(\mathrm{C}_{8} \mathrm{~F}_{4} \mathrm{O}_{4}\right)^{2-}\end{array}$ & $\begin{array}{c}\text { bis(4,4'-Bipyridinium) } 2,3,5,6- \\
\text { tetrafluoroterephthalate } 2,3,5,6- \\
\text { tetrafluoroterephthalic acid }\end{array}$ & 0.5 & 3 & 3 & CAB \\
\hline 80 & VADVOM & $\begin{array}{c}2\left(\mathrm{C}_{14} \mathrm{H}_{19} \mathrm{~N}_{4} \mathrm{O}_{3}\right)^{1+},\left(\mathrm{C}_{8} \mathrm{H}_{4} \mathrm{O}_{4}\right)^{2-} \\
\left(\mathrm{C}_{8} \mathrm{H}_{6} \mathrm{O}_{4}\right)\end{array}$ & $\begin{array}{c}\text { 2,4-Diamino-5-(3,4,5- } \\
\text { trimethoxybenzyl)pyrimidin-1-ium terephthalate } \\
\text { terephthalic acid }\end{array}$ & 0.5 & 3 & 3 & $C A B$ \\
\hline 81 & VAQTUD & $\begin{array}{l}\left(\mathrm{C}_{5} \mathrm{H}_{8} \mathrm{~N}_{3}\right)^{1+},\left(\mathrm{C}_{5} \mathrm{H}_{8} \mathrm{~N}_{3}\right)^{1+}, \\
\left(\mathrm{C}_{5} \mathrm{H}_{5} \mathrm{O}_{4}\right)^{1-},\left(\mathrm{C}_{5} \mathrm{H}_{5} \mathrm{O}_{4}\right)^{1-}\end{array}$ & $\begin{array}{l}\text { 2-Amino-4-methylpyrimidin-1-ium 2- } \\
\text { amino-4-methylpyrimidin-3-ium 2-methyl } \\
\text { hydrogen maleate 3-methyl hydrogen maleate }\end{array}$ & 1 & 4 & 4 & $C A B$ \\
\hline 82 & VARGAZ & $\begin{array}{l}\left(\mathrm{C}_{12} \mathrm{H}_{11} \mathrm{Cl}_{1} \mathrm{~N}_{2} \mathrm{O}_{5} \mathrm{~S}_{1}\right) \\
\left(\mathrm{C}_{12} \mathrm{H}_{10} \mathrm{Cl}_{1} \mathrm{~N}_{2} \mathrm{O}_{5} \mathrm{~S}_{1}\right)^{1-} \\
\left(\mathrm{C}_{12} \mathrm{H}_{11} \mathrm{~N}_{2}\right)^{1+}\end{array}$ & $\begin{array}{l}\text { 4-[2-(pyridin-4-yl)ethenyl]pyridin-1-ium 4- } \\
\text { chloro-2-\{[(furan-2-yl)methyl]amino\}-5- } \\
\text { sulfamoylbenzoate 4-chloro-2-\{[(furan-2- } \\
\text { yl)methyl]amino\}-5-sulfamoylbenzoic acid }\end{array}$ & 1 & 3 & 3 & $\mathrm{CAB}$ \\
\hline 83 & VOKCOQ & $\begin{array}{c}\left(\mathrm{C}_{21} \mathrm{H}_{14} \mathrm{Br}_{1} \mathrm{O}_{4} \mathrm{~S}_{2}\right)^{1-} \\
\left(\mathrm{C}_{5} \mathrm{H}_{6} \mathrm{~N}_{1}\right)^{1+},\left(\mathrm{C}_{5} \mathrm{H}_{5} \mathrm{~N}_{1}\right)\end{array}$ & $\begin{array}{c}\text { pyridin-1-ium pyridine 2-(\{(2- } \\
\text { bromophenyl)[(2- } \\
\text { carboxyphenyl)sulfanyl]methyl\}sulfanyl)benzoate }\end{array}$ & 1 & 3 & 3 & $\mathrm{CAB}$ \\
\hline 84 & VUKPAU & $\begin{array}{c}\left(\mathrm{C}_{8} \mathrm{H}_{12} \mathrm{~N}_{1} \mathrm{O}_{3}\right)^{1+},\left(\mathrm{C}_{7} \mathrm{H}_{5} \mathrm{~N}_{1} \mathrm{O}_{4}\right), \\
\left(\mathrm{C}_{7} \mathrm{H}_{4} \mathrm{~N}_{1} \mathrm{O}_{4}\right)^{1-}\end{array}$ & $\begin{array}{l}\text { 3-hydroxy-4,5-bis(hydroxymethyl)-2- } \\
\text { methylpyridin-1-ium 4-nitrobenzoic acid 4- } \\
\text { nitrobenzoate }\end{array}$ & 1 & 3 & 3 & $\mathrm{CAB}$ \\
\hline 85 & WADPAU & $\begin{array}{c}\left(\mathrm{C}_{10} \mathrm{H}_{9} \mathrm{~N}_{2}\right)^{1+},\left(\mathrm{C}_{8} \mathrm{H}_{3} \mathrm{I}_{3} \mathrm{~N}_{1} \mathrm{O}_{4}\right)^{1-}, \\
\left(\mathrm{C}_{8} \mathrm{H}_{4} \mathrm{I}_{3} \mathrm{~N}_{1} \mathrm{O}_{4}\right)\end{array}$ & $\begin{array}{l}\text { 4-(4-Pyridyl)pyridinium 3-amino-5- } \\
\text { carboxy-2,4,6-triiodobenzoate 5-amino-2,4,6-tri- } \\
\text { iodoisophthalic acid }\end{array}$ & 1 & 3 & 3 & CAB \\
\hline
\end{tabular}




\begin{tabular}{|c|c|c|c|c|c|c|c|}
\hline 86 & WAQMAD & $\begin{array}{c}2\left(\mathrm{C}_{10} \mathrm{H}_{9} \mathrm{~N}_{2}\right)^{1+},\left(\mathrm{C}_{10} \mathrm{H}_{4} \mathrm{O}_{8}\right)^{2-} \\
\left(\mathrm{C}_{10} \mathrm{H}_{6} \mathrm{O}_{8}\right)\end{array}$ & $\begin{array}{c}\text { bis(2,4'-Bipyridin-1'-ium) 2,5- } \\
\text { dicarboxybenzene-1,4-carboxylate benzene- } \\
\text { 1,2,4,5-tetracarboxylic acid }\end{array}$ & 0.5 & 3 & 3 & $\mathrm{CAB}$ \\
\hline 87 & WEVXED & $\begin{array}{c}\left(\mathrm{C}_{16} \mathrm{H}_{6} \mathrm{~N}_{2} \mathrm{O}_{12}\right)^{2-} \\
2\left(\mathrm{C}_{13} \mathrm{H}_{10} \mathrm{~N}_{1}\right)^{1+},\left(\mathrm{C}_{14} \mathrm{H}_{10}\right)\end{array}$ & $\begin{array}{l}\text { bis(acridin-10-ium) phenanthrene 2',6'- } \\
\text { dicarboxy-4,4'-dinitro[1,1'-biphenyl]-2,6- } \\
\text { dicarboxylate }\end{array}$ & 1 & 3 & 3 & NCAB \\
\hline 88 & WEVXIH & $\begin{array}{c}\left(\mathrm{C}_{16} \mathrm{H}_{6} \mathrm{~N}_{2} \mathrm{O}_{12}\right)^{2-}, 2\left(\mathrm{C}_{15} \mathrm{H}_{10} \mathrm{O}_{1}\right) \\
2\left(\mathrm{C}_{13} \mathrm{H}_{10} \mathrm{~N}_{1}\right)^{1+}\end{array}$ & $\begin{array}{l}\text { bis(acridin-10-ium) bis(anthracene-9- } \\
\text { carbaldehyde) 2',6'-dicarboxy-4,4'-dinitro[1,1'- } \\
\text { biphenyl]-2,6-dicarboxylate }\end{array}$ & 1 & 3 & 3 & $\mathrm{NCAB}$ \\
\hline 89 & WOVBIV & $\begin{array}{c}2\left(\mathrm{C}_{13} \mathrm{H}_{10} \mathrm{~N}_{1}\right)^{1+},\left(\mathrm{C}_{8} \mathrm{H}_{6} \mathrm{O}_{6}\right) \\
\left(\mathrm{C}_{8} \mathrm{H}_{4} \mathrm{O}_{6}\right)^{2-}\end{array}$ & $\begin{array}{l}\text { bis(acridin-10-ium) } 2,5- \\
\text { dihydroxyterephthalate } 2,5- \\
\text { dihydroxyterephthalic acid }\end{array}$ & 0.5 & 3 & 3 & $\mathrm{CAB}$ \\
\hline 90 & XAQPAI & $\begin{array}{c}2\left(\mathrm{C}_{8} \mathrm{H}_{12} \mathrm{~N}_{1} \mathrm{O}_{1}\right)^{1+},\left(\mathrm{C}_{4} \mathrm{H}_{4} \mathrm{O}_{4}\right)^{2-} \\
\left(\mathrm{C}_{4} \mathrm{H}_{6} \mathrm{O}_{4}\right)\end{array}$ & $\begin{array}{c}\text { bis(2-Ethyl-6-methyl-3- } \\
\text { hydroxypyridinium) succinate succinic acid }\end{array}$ & 0.5 & 3 & 3 & $C A B$ \\
\hline 91 & XINRUH & $\begin{array}{c}2\left(\mathrm{C}_{5} \mathrm{H}_{7} \mathrm{~N}_{2}\right)^{+},\left(\mathrm{C}_{4} \mathrm{H}_{2} \mathrm{O}_{4}\right)^{2-} \\
\left(\mathrm{C}_{4} \mathrm{H}_{4} \mathrm{O}_{4}\right)\end{array}$ & $\begin{array}{l}\text { bis(2-Aminopyridinium) fumarate fumaric } \\
\text { acid }\end{array}$ & 0.5 & 3 & 3 & $C A B$ \\
\hline 92 & XIPTUM & $\begin{array}{l}\left(\mathrm{C}_{8} \mathrm{H}_{7} \mathrm{~N}_{2}\right)^{1+},\left(\mathrm{C}_{8} \mathrm{H}_{6} \mathrm{~N}_{2}\right) \\
\left(\mathrm{C}_{7} \mathrm{H}_{5} \mathrm{O}_{5}\right)^{1-},\left(\mathrm{C}_{7} \mathrm{H}_{6} \mathrm{O}_{5}\right)\end{array}$ & $\begin{array}{c}\text { Quinoxalinium quinoxaline } 2,4,6- \\
\text { trihydroxybenzoate } 2,4,6 \text {-trihydroxybenzoic acid }\end{array}$ & 1 & 4 & 4 & $C A B$ \\
\hline 93 & XONCAG & $\begin{array}{c}\left(\mathrm{C}_{13} \mathrm{H}_{16} \mathrm{~N}_{2}\right)^{2+},\left(\mathrm{C}_{6} \mathrm{H}_{4} \mathrm{O}_{4} \mathrm{~S}_{1}\right) \\
\left(\mathrm{C}_{6} \mathrm{H}_{2} \mathrm{O}_{4} \mathrm{~S}_{1}\right)^{2-}\end{array}$ & $\begin{array}{c}\text { 4,4'-propane-1,3-diyldipyridin-1-ium } \\
\text { thiophene-2,5-dicarboxylic acid thiophene-2,5- } \\
\text { dicarboxylate }\end{array}$ & 0.5 & 3 & 3 & $C A B$ \\
\hline 94 & ZAJHUN & $\begin{array}{c}\left(\mathrm{C}_{4} \mathrm{H}_{6} \mathrm{~N}_{3}\right)^{1+},\left(\mathrm{C}_{7} \mathrm{H}_{4} \mathrm{~N}_{1} \mathrm{O}_{4}\right)^{1-} \\
\left(\mathrm{C}_{7} \mathrm{H}_{5} \mathrm{~N}_{1} \mathrm{O}_{4}\right)\end{array}$ & $\begin{array}{l}\text { 2-Aminopyrimidine bis(2-nitrobenzoic } \\
\text { acid) }\end{array}$ & 1 & 3 & 3 & $C A B$ \\
\hline
\end{tabular}

Table S1: Summary of the 94 molecular ICCs retrieved from the CSD and their structural properties. The Ncu column contains the calculated order of the molecular ICC using the proposed equation 5 in the main text and is consistent with the CSD "chemical units" entry for each ICC. * In these crystals, conjugate acid-base pairs do exist but the crystal also contains chemically distinct coformers and as such, the solid form is classified as an "NCAB" ICC. 


\section{Calculated Molecular and Crystal Properties}

\subsection{Table S2: Molecular Properties}

\begin{tabular}{|c|c|c|c|c|c|c|}
\hline ICC Refcode* & $\Delta p K_{a}$ & $\begin{array}{c}\text { Acid Polar } \\
\text { Surface Area } \\
\left(\AA^{2}\right)\end{array}$ & $\begin{array}{c}\text { Base Polar } \\
\text { Surface Area } \\
\left(\AA^{2}\right)\end{array}$ & $\begin{array}{c}\text { Acid Number of } \\
\text { Conformers }\end{array}$ & $\begin{array}{c}\text { Base Number of } \\
\text { Conformers }\end{array}$ & $\begin{array}{c}\text { Difference in } \\
\text { Acid-Base } \\
\text { Conformers }\end{array}$ \\
\hline AFORIY01 & 7.6 & 128.94 & 38.91 & 7 & 1 & 6 \\
\hline AJEBIA & 3.13 & 149.17 & 12.89 & 7 & 1 & 6 \\
\hline APURAG & 2.17 & 83.12 & 12.89 & 1 & 1 & 0 \\
\hline AYUSIX & 3.85 & 83.12 & 12.89 & 1 & 1 & 0 \\
\hline CAYJAO01 & 1.82 & 128.94 & 12.89 & 1 & 1 & 0 \\
\hline CIDCUO & 2.97 & 115.06 & 70.26 & 16 & 3 & 13 \\
\hline CUCSEY01 & 3.08 & 37.3 & 105.51 & 1 & 200 & -199 \\
\hline CUCSEY10 & 3.08 & 37.3 & 105.51 & 1 & 200 & -199 \\
\hline CUKNON & 4.71 & 37.3 & 16.13 & 1 & 1 & 0 \\
\hline CUKNUT & 4.4 & 57.53 & 16.13 & 1 & 1 & 0 \\
\hline DEFCUM & 3.76 & 74.6 & 12.89 & 4 & 1 & 3 \\
\hline DEMYAV & 2.38 & 74.6 & 50.28 & 4 & 200 & -196 \\
\hline DICYIA & 3.29 & 74.6 & 38.91 & 42 & 1 & 41 \\
\hline DUPJEE & 3.32 & 37.3 & 64.93 & 1 & 1 & 0 \\
\hline DUTNUC & 3.87 & 74.6 & 38.91 & 3 & 1 & 2 \\
\hline DUVLUB & 3.76 & 74.6 & 12.89 & 4 & 1 & 3 \\
\hline ECESAJ & 1.67 & 86.63 & 12.89 & 145 & 1 & 144 \\
\hline EMUMIK & 2.87 & 74.6 & 12.89 & 9 & 1 & 8 \\
\hline EYIWOY & 8.03 & 111.9 & 50.19 & 16 & 2 & 14 \\
\hline EZEGOH & 2.54 & 83.12 & 38.91 & 2 & 12 & -10 \\
\hline
\end{tabular}




\begin{tabular}{|c|c|c|c|c|c|c|}
\hline FEBSOX & 1.13 & 83.12 & 55.98 & 6 & 1 & 5 \\
\hline FECGIG & 1.31 & 83.12 & 55.98 & 7 & 2 & 5 \\
\hline FIBHOP & 1.55 & 74.6 & 25.78 & 3 & 10 & -7 \\
\hline GEPFIR & 6.48 & 37.3 & 16.13 & 4 & 1 & 3 \\
\hline GIQHUM & 2.65 & 37.3 & 54.02 & 1 & 97 & -96 \\
\hline GUKWIT & 5.43 & 74.6 & 16.13 & 3 & 1 & 2 \\
\hline GULRAJ & 2.75 & 101.54 & 25.78 & 8 & 1 & 7 \\
\hline HAXFER & 2.84 & 128.94 & 12.89 & 1 & 1 & 0 \\
\hline HIFZUU & 2.41 & 37.3 & 38.91 & 14 & 1 & 13 \\
\hline IGOGES & 1.63 & 74.6 & 41.57 & 2 & 2 & 0 \\
\hline IHESOD01 & 3.29 & 74.6 & 38.91 & 42 & 1 & 41 \\
\hline IXOZOL & 6.1 & 74.6 & 38.91 & 12 & 1 & 11 \\
\hline JAWVUZ & 0.73 & 115.06 & 55.98 & 15 & 1 & 14 \\
\hline JEJNAO & 0 & 128.94 & 25.78 & 1 & 2 & -1 \\
\hline JOYJIS & -0.14 & 74.6 & 51.8 & 107 & 1 & 106 \\
\hline KALQUM & 0.25 & 94.83 & 55.98 & 48 & 1 & 47 \\
\hline KEKHAK & 1.84 & 74.68 & 33.12 & 6 & 1 & 5 \\
\hline KOXREU & 2.69 & 74.6 & 12.89 & 9 & 1 & 8 \\
\hline KUJDIE & 5.99 & 57.53 & 16.13 & 4 & 1 & 3 \\
\hline KUZJIZ & 1.87 & 74.6 & 38.91 & 8 & 1 & 7 \\
\hline LAPNUM & 3.3 & 74.6 & 38.91 & 200 & 1 & 199 \\
\hline LAPPAU & 4.25 & 74.6 & 38.91 & 3 & 1 & 2 \\
\hline LATLID & 0.58 & 83.12 & 54.35 & 2 & 4 & -2 \\
\hline LOCMUM & 2.83 & 149.2 & 25.78 & 65 & 1 & 64 \\
\hline MELZAF & 2.83 & 149.2 & 25.78 & 65 & 1 & 64 \\
\hline MEXDAW & 2.97 & 74.6 & 38.91 & 9 & 1 & 8 \\
\hline MUFJUV & 5.71 & 37.3 & 16.13 & 4 & 1 & 3 \\
\hline MUYSOP01 & 2.4 & 62.22 & 62.22 & 22 & 22 & 0 \\
\hline
\end{tabular}




\begin{tabular}{|c|c|c|c|c|c|c|}
\hline NIMDIW & 2.04 & 57.53 & 33.12 & 4 & 1 & 3 \\
\hline OJUJUA & 2.56 & 37.3 & 38.91 & 1 & 1 & 0 \\
\hline OVUMEY & 1.67 & 37.3 & 90.71 & 1 & 4 & -3 \\
\hline PEKSUV & 2.72 & 77.76 & 25.78 & 4 & 3 & 1 \\
\hline PIWXEA & 1.76 & 74.6 & 95.17 & 200 & 200 & 0 \\
\hline QABZAX & 0.63 & 37.3 & 51.56 & 1 & 1 & 0 \\
\hline QAFFOS & 0.85 & 37.3 & 12.89 & 1 & 1 & 0 \\
\hline QAFFOSO1 & 0.85 & 37.3 & 12.89 & 1 & 1 & 0 \\
\hline RENVUE & 2.22 & 86.63 & 12.89 & 143 & 1 & 142 \\
\hline RENWAL & 2.26 & 86.63 & 12.89 & 142 & 1 & 141 \\
\hline RESFUR & 3.23 & 74.6 & 12.89 & 3 & 1 & 2 \\
\hline RESGEC & 2.89 & 74.6 & 12.89 & 3 & 1 & 2 \\
\hline RESGUS & 2.98 & 74.6 & 12.89 & 3 & 1 & 2 \\
\hline REXNOZ & 7.6 & 74.6 & 16.13 & 6 & 1 & 5 \\
\hline ROBFAS & 3.25 & 37.3 & 77.82 & 2 & 6 & -4 \\
\hline ROFCEV & 5.49 & 83.12 & 38.91 & 1 & 1 & 0 \\
\hline SAGWII & 2.62 & 37.3 & 64.93 & 4 & 1 & 3 \\
\hline SEGSAZ & 4.12 & 74.6 & 41.05 & 3 & 200 & -197 \\
\hline TAKZUC & 3.37 & 57.53 & 38.91 & 6 & 1 & 5 \\
\hline TEKLOK & 1.33 & 149.2 & 25.78 & 61 & 2 & 59 \\
\hline TICZOW & 1.78 & 128.94 & 12.89 & 1 & 3 & -2 \\
\hline UNECEV & 6.81 & 149.2 & 16.13 & 66 & 1 & 65 \\
\hline UVOKIA & 5.64 & 74.6 & 38.91 & 3 & 1 & 2 \\
\hline UYOZEO & 4.07 & 74.6 & 25.78 & 6 & 3 & 3 \\
\hline VADVOM & 3.84 & 74.6 & 105.51 & 2 & 200 & -198 \\
\hline VAQTUD & 2.22 & 74.6 & 51.8 & 17 & 1 & 16 \\
\hline VARGAZ & 1.34 & 122.63 & 25.78 & 200 & 1 & 199 \\
\hline VOKCOQ & 2.05 & 74.6 & 12.89 & 200 & 1 & 199 \\
\hline
\end{tabular}




\begin{tabular}{|c|c|c|c|c|c|c|}
\hline VUKPAU & 2.12 & 83.12 & 73.58 & 1 & 7 & -6 \\
\hline WADPAU & 2.6 & 100.62 & 25.78 & 3 & 3 & 0 \\
\hline WAQMAD & 1.75 & 149.2 & 25.78 & 65 & 1 & 64 \\
\hline WOVBIV & 4.35 & 115.06 & 12.89 & 9 & 1 & 8 \\
\hline XAQPAI & 2.94 & 74.6 & 33.12 & 42 & 3 & 39 \\
\hline XINRUH & 3.49 & 74.6 & 38.91 & 3 & 1 & 2 \\
\hline XIPTUM & -0.09 & 97.99 & 25.78 & 2 & 1 & 1 \\
\hline XONCAG & 3.05 & 74.6 & 25.78 & 3 & 162 & -159 \\
\hline ZAJHUN & 1.3 & 83.12 & 51.8 & 7 & 1 & 6 \\
\hline
\end{tabular}

Table S2: Calculated $\Delta p K_{a}$ and Polar Surface Areas for the molecular ICCs shown. * For redeterminations of some crystal structures, we kept only the best determination of the crystal as determined by the R-factor. For all other crystals that may contain the same acid-base pairs including polymorphs, since the crystal structures are distinct, the resulting molecular property data was kept. 
2.2 Figure S2: Molecular structures for the most common acidic molecules used to crystallize molecular ICCs

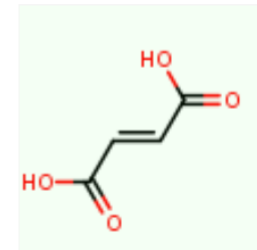<smiles>O=C(O)c1cc([N+](=O)[O-])cc([N+](=O)[O-])c1</smiles><smiles>O=C(O)c1cc(C(=O)O)c(C(=O)O)cc1C(=O)O</smiles><smiles>O=C(O)c1ccccc1O</smiles><smiles>O=C(O)c1ccc([N+](=O)[O-])cc1</smiles><smiles>O=C(O)c1ccccc1</smiles><smiles>O=C(O)CCC(=O)O</smiles><smiles>O=C(O)C(=O)O</smiles><smiles>O=C(O)CNc1ccccc1C(=O)O</smiles><smiles>O=C(O)c1ccccc1[N+](=O)[O-]</smiles><smiles>O=C(O)C(O)C(O)C(=O)O</smiles><smiles>O=C(O)c1ccc(Cl)cc1</smiles><smiles>O=C(O)c1ccc(O)cc1</smiles><smiles>O=C(O)c1ccccc1C(=O)O</smiles><smiles>O=C(O)c1ccc([N+](=O)[O-])cc1Cl</smiles><smiles>O=C(O)C(F)(F)F</smiles><smiles>O=C(O)c1ccc(C(=O)O)cc1</smiles><smiles>O=C(O)CC(=O)O</smiles><smiles>O=C(O)c1ccc(C(=O)O)s1</smiles><smiles>O=C(O)c1c2ccccc2cc2ccccc12</smiles><smiles>O=C(O)c1c(F)c(F)c(C(=O)O)c(F)c1F</smiles><smiles>O=CO</smiles><smiles>O=C(O)c1cc([N+](=O)[O-])cc(C(=O)O)c1-c1c(C(=O)O)cc([N+](=O)[O-])cc1C(=O)O</smiles>

Figure S2: Molecular structures for the most frequently recurring acid molecules used to crystallize the molecular ICCs listed in Table S1. The green highlighting shows the two most frequently used acid molecules. 
2.3 Figure S3: Molecular structures for the most common basic molecules used to crystallize molecular ICCS<smiles>CN(C)c1ccncc1</smiles><smiles>c1ccncc1</smiles><smiles>c1cc(-c2ccncc2)ccn1</smiles><smiles>Nc1ccccn1</smiles><smiles>NC(=O)c1ccncc1</smiles><smiles>Nc1ccncc1</smiles><smiles>c1cnc2c(c1)ccc1cccnc12</smiles><smiles>COc1cc(Cc2cnc(N)nc2N)cc(OC)c1OC</smiles><smiles>c1ccc(-c2ccccn2)nc1</smiles><smiles>Cc1ccc(N)nc1</smiles><smiles>c1ccc2nc3ccccc3cc2c1</smiles><smiles>Cc1cccnc1</smiles><smiles>Nc1cccnc1N</smiles><smiles>Nc1ncccn1</smiles><smiles>Oc1cccc2cccnc12</smiles>

Figure S3: Molecular structures for the most frequently recurring basic molecules used to crystallize the molecular ICCs listed in Table S1. The red highlighting shows the two most frequently used basic molecules. 


\subsection{Table S3: Periodic DFT-D stabilization energies for the molecular ICCs}

\begin{tabular}{|c|c|c|c|c|c|c|c|}
\hline \multicolumn{3}{|c|}{ ICC } & \multicolumn{2}{|r|}{ Salt } & \multicolumn{2}{|c|}{ Coformer } & \multirow{2}{*}{$\begin{array}{c}\Delta E_{I C C} \\
(\mathrm{~kJ} / \mathrm{mol})\end{array}$} \\
\hline Refcode & Volume $\left(\AA^{3}\right)$ & $\begin{array}{c}\text { Energy per mole } \\
(\mathrm{eV})\end{array}$ & Refcode & $\begin{array}{c}\text { Energy per mole } \\
(\mathrm{eV})\end{array}$ & Refcode & $\begin{array}{c}\text { Energy per mole } \\
(\mathrm{eV})\end{array}$ & \\
\hline CUCSEY10 & 1299.73 & -449.11 & CESRUN & -348.61 & BENZAC12 & -100.36 & -13.45 \\
\hline CUKNON & 1026.52 & -315.94 & CUKNED & -216.79 & CLBZAP03 & -99.11 & -3.47 \\
\hline CUKNUT & 964.86 & -332.82 & SOLGUX01 & -225.34 & JOZZIH01 & -107.42 & -6.02 \\
\hline DUPJEE & 1740.89 & -298.4 & BOVTEM & -198.10 & BENZAC12 & -100.36 & 5.69 \\
\hline KUHTUE & 935.63 & -325.78 & KUJDEA & -225.38 & BENZAC12 & -100.36 & -4.43 \\
\hline KUHVAM & 961.17 & -324.53 & KUJDEA & -225.38 & CLBZAP03 & -99.11 & -3.5 \\
\hline KUHVEQ & 1917.47 & -332.95 & KUJDEA & -225.38 & JOZZIH01 & -107.42 & -14.16 \\
\hline KUJDIE & 1932.35 & -332.84 & KUJDEA & -225.38 & SALIAC16 & -107.43 & -2.38 \\
\hline MUFJUV & 1014.38 & -315.57 & MUFKEG & -216.68 & CLBZAC03 & -98.88 & -1.12 \\
\hline NIMDIW & 1004.95 & -338.44 & HURNEN & -230.98 & SALIAC16 & -107.43 & -2.65 \\
\hline SAGWII & 1742.83 & -293.94 & SUZSOW & -195.82 & ZZZNWQ02 & -98.19 & 6.65 \\
\hline WEVXED & 2187.85 & -756.52 & WEVWUS & -591.35 & PHENAN08 & -165.10 & -7.67 \\
\hline WEVXIH & 5468.85 & -952.11 & WEVWUS & -591.35 & ANTHALO2 & -180.28 & -19.32 \\
\hline XAQPAI & 660.25 & -434.99 & BECYIT & -347.33 & SUCACB03 & -87.68 & 1.72 \\
\hline
\end{tabular}

Table S3: Electronic structure energies for the crystal structures listed following full geometry optimization (cell and atom positions) of the crystals using VASP. The molecular ICC stabilization energy $\left(\triangle E_{I C C}\right)$ was estimated according to equation 6 shown in the manuscript. 
2.5 Table S4: Calculated changes in the crystal packing index upon switching from the salt to the ICC

\begin{tabular}{|c|c|c|c|r|}
\hline ICC Refcode & $\boldsymbol{C}_{\boldsymbol{K}}($ ICC, $\%)$ & Salt Refcode & $\begin{array}{c}\boldsymbol{C}_{\boldsymbol{K}} \\
(\text { Salt, } \%)\end{array}$ & $\Delta \boldsymbol{C}_{\boldsymbol{K}}(\%)$ \\
\hline CUCSEY10 & 72.6 & CESRUN & 71.4 & 1.2 \\
\hline CUKNON & 71.6 & CUKNED & 72 & -0.4 \\
\hline CUKNUT & 73.2 & SOLGUX01 & 73.1 & 0.1 \\
\hline DUPJEE & 72.2 & BOVTEM & 70.5 & 1.7 \\
\hline KUHTUE & 74.2 & KUJDEA & 72.4 & 1.8 \\
\hline KUHVAM & 74.9 & KUJDEA & 72.4 & 2.5 \\
\hline KUHVEQ & 73.6 & KUJDEA & 72.4 & 1.2 \\
\hline KUJDIE & 73.2 & KUJDEA & 72.4 & 0.8 \\
\hline MUFJUV & 72.5 & MUFKEG & 73 & -0.5 \\
\hline NIMDIW & 71.2 & HURNEN & 72.2 & -1 \\
\hline SAGWII & 72.2 & SUZSOW & 68.4 & 3.8 \\
\hline WEVXED & 73.5 & WEVWUS & 73.1 & 0.4 \\
\hline WEVXIH & 73.9 & WEVWUS & 73.1 & 0.8 \\
\hline XAQPAI & 69.9 & BECYIT & 72.2 & -2.3 \\
\hline
\end{tabular}

Table S4: Calculated changes in the packing index of the crystal upon switching from the salt to the molecular ICC. 
2.6 Table S5: Common motifs found in the molecular ICCs

\begin{tabular}{|c|c|c|}
\hline Crystal Index & ICC Refcode & Synthon Type \\
\hline 1 & AFORIY01 & Type 2 \\
\hline 2 & AJEBIA & Type 2 \\
\hline 3 & APURAG & Type 1 \\
\hline 4 & AYUSIX & Type 1 \\
\hline 5 & BEYZAI & Type 1 \\
\hline 6 & BEYZIQ & Type 1 \\
\hline 7 & CAYJAO01 & Type 2 \\
\hline 8 & CIDCUO & Other \\
\hline 9 & CUCSEY01 & Type 1 \\
\hline 10 & CUCSEY10 & Type 2 \\
\hline 11 & CUKNON & Type 1 \\
\hline 12 & CUKNUT & Type 3 \\
\hline 13 & DEFCUM & Type 1 \\
\hline 14 & DEMYAV & Other \\
\hline 15 & DICYIA & Type 2 \\
\hline 16 & DUPJEE & Type 1 \\
\hline 17 & DUTNUC & Type 2 \\
\hline 18 & DUVLUB & Type 3 \\
\hline
\end{tabular}




\begin{tabular}{|c|c|c|}
\hline 19 & ECESAJ & Type 2 \\
\hline 20 & EMUMIK & Other \\
\hline 21 & EYIWOY & Type 1 \\
\hline 22 & EZEGOH & Other \\
\hline 23 & FEBSOX & Other \\
\hline 24 & FECGIG & Type 1 \\
\hline 25 & FIBHOP & Type 1 \\
\hline 26 & GEPFIR & Type 1 \\
\hline 27 & GIQHUM & Type 3 \\
\hline 28 & GUKWIT & Type 2 \\
\hline 29 & GULRAJ & Type 1 \\
\hline 30 & HAXFER & Type 1 \\
\hline 31 & HIFZUU & Type 2 \\
\hline 32 & IGOGES & Other \\
\hline 33 & IHESOD01 & Type 2 \\
\hline 34 & IXOZOL & Other \\
\hline 35 & JAWVUZ & Other \\
\hline 36 & JEJNAO & Type 1 \\
\hline 37 & JOYJIS & Type 2 \\
\hline 38 & KALQUM & Other \\
\hline 39 & KEKHAK & Type 2 \\
\hline
\end{tabular}




\begin{tabular}{|c|c|c|}
\hline 40 & KOXREU & Type 1 \\
\hline 41 & KUHTUE & Type 1 \\
\hline 42 & KUHVAM & Type 1 \\
\hline 43 & KUHVEQ & Type 1 \\
\hline 44 & KUJDIE & Type 1 \\
\hline 45 & KUZJIZ & Other \\
\hline 46 & LAPNUM & Type 2 \\
\hline 47 & LAPPAU & Type 1 \\
\hline 48 & LATLID & Other \\
\hline 49 & LOCMUM & Other \\
\hline 50 & LORWOF & Other \\
\hline 51 & MELZAF & Other \\
\hline 52 & MEXDAW & Type 2 \\
\hline 53 & MUFJUV & Type 2 \\
\hline 54 & MUYSOP01 & Type 1 \\
\hline 55 & NEWNIP & Type 1 \\
\hline 56 & NIMDIW & Type 3 \\
\hline 57 & OJUJUA & Type 1 \\
\hline 58 & OVUMEY & Other \\
\hline 59 & PEKSUV & Other \\
\hline 60 & PIWXEA & Type 2 \\
\hline
\end{tabular}




\begin{tabular}{|c|c|c|}
\hline 61 & QABZAX & Type 1 \\
\hline 62 & QAFFOS & Type 3 \\
\hline 63 & QAFFOSO1 & Type 3 \\
\hline 64 & RENVUE & Type 2 \\
\hline 65 & RENWAL & Type 3 \\
\hline 66 & RESFUR & Type 1 \\
\hline 67 & RESGEC & Type 1 \\
\hline 68 & RESGUS & Type 1 \\
\hline 69 & REXNOZ & Type 1 \\
\hline 70 & ROBFAS & Type 1 \\
\hline 71 & ROFCEV & Type 3 \\
\hline 72 & SAGWII & Other \\
\hline 73 & SEGSAZ & Type 3 \\
\hline 74 & TAKZUC & Type 1 \\
\hline 75 & TEKLOK & Type 3 \\
\hline 76 & TICZOW & Type 1 \\
\hline 77 & UNECEV & Type 3 \\
\hline 78 & UVOKIA & Type 2 \\
\hline 79 & UYOZEO & Other \\
\hline 80 & VADVOM & Type 1 \\
\hline 81 & VAQTUD & Other \\
\hline
\end{tabular}




\begin{tabular}{|c|c|c|}
\hline 82 & VARGAZ & Other \\
\hline 83 & VOKCOQ & Other \\
\hline 84 & VUKPAU 1 \\
\hline 85 & WADPAU & Type 1 \\
\hline 86 & WAQMAD & Other \\
\hline 87 & WEVXED 2 \\
\hline 88 & WEVXIH & Type 1 \\
\hline 89 & WOVBIV & Type 1 \\
\hline 90 & XAQPAI & Type 2 2 \\
\hline 91 & XINRUH & Other \\
\hline 92 & XIPTUM & Type 1 \\
\hline 93 & XONCAG & Type 1 \\
\hline 94 & ZAJHUN & \\
\hline
\end{tabular}

Table S5: Classification of the most common supramolecular heterosynthons found in the molecular ICCs. Other= ICCs not showing any of the common synthons labelled Types 1-3. 